\title{
Intervención psicomotriz en el área personal/social de un grupo de educación preescolar
}

\section{Psychomotor intervention on social/personal area of a group of children of pre-school education}

\author{
Rocío RUBIO PONCE; David Arnoldo GARCÍA FERNÁNDEZ; \\ Natanael CERVANTES HERNÁNDEZ
}

Facultad de Educación Física y Ciencias del Deporte

Universidad Autónoma de Chihuahua

Recibido: Marzo 2011

Aceptado: Diciembre 2011

\section{Resumen}

En el presente estudio se realiza el análisis de un grupo de niños y niñas de tercer grado de educación preescolar, en cuanto a la posible mejoría en su área personal/social que abarca la interacción con el adulto, autoconcepto, interacción con los compañeros, colaboración y rol social a través de la aplicación de un programa de intervención psicomotriz con base en el juego, utilizando música de fondo y material reciclado. El grupo experimental integrado por 29 alumnos (13 niños y 16 niñas), se sometió a una evaluación pre-post, utilizándose como instrumento de medición el área personal/social del Inventario de Desarrollo Battelle y a un programa psicomotriz con una duración de tres meses en 36 sesiones. En la comparación de resultados de evaluación previos y posteriores al programa, interpretados a través de estadística descriptiva, prueba t de Student y regresión lineal, se observaron diferencias positivas y significativas después de la intervención.

Palabras clave: programa psicomotriz, área personal/social, intervención en preescolar, interacción con el adulto, autoconcepto, interacción con los compañeros, colaboración, rol social.

\begin{abstract}
In the present study there are realized the analysis of a group of children of the third degree of pre-school education, as for the possible improvement in their personal / social area that includes the interaction with the adult, autoconcept, interaction with the companions, collaboration and social role across the application of a program of psychomotor intervention with base in the game, using background music and recycled material. The experimental group integrated by 29 pupils (13 boys and 16 girls), surrendered to an evaluation pre-post, being in use as instrument of measurement the personal / social area of the Inventory of Development Battelle
\end{abstract}


and to a psychomotor program with a duration of three months in 36 meetings. In the comparison of results of evaluation before and later to the program, interpreted across descriptive statistics, it tries t of student and linear regression, were observed positive and significant differences after the intervention.

Keywords: psychomotor program, social/personal area, intervention in pre-school, interaction with the adult, auto concept, interaction with the companions, collaboration, social role.

Hoy en día la educación preescolar actual introduce planeamientos generales sobre sus objetivos divididos por competencias, donde se determinan y planifican actividades concretas en relación a los campos formativos que competen a los niños de la etapa infantil temprana, pero a nivel práctico, cuando se trata de descender de las formulaciones teóricas al proceso de educación y dar un sentido, al desarrollo operativo de instrucción, tales principios apenas encuentran reflejo. Es por esta razón que se hace necesario el uso de modelos integradores como la psicomotricidad relacional (Zabalza, 2006).

El concepto personalidad se utiliza para transmitir un sentido de coherencia y continuidad en el individuo, para comunicar la idea de que en la persona está el origen de la conducta en la que está ocupada y para manifestar la idea de que la esencia del sujeto puede ser resumida o captada en cualidades destacables que dan forma y continuidad a la conducta (Craver y Sheier, 1997).

Newborg, Stock y Wnek (1998) describen el área personal/social como la que supone las características que permiten al niño establecer relaciones significativas, las conductas apreciadas en ésta área, se agrupan en seis subáreas:

a) Interacción con el adulto: Evalúa la calidad y frecuencia de las interacciones del niño con los adultos. Estas interacciones incluyen comportamientos como el apego infantil, la respuesta al inicio de contactos sociales y la utilización de los adultos como recurso.

b) Expresión de sentimientos/afecto: Evalúa la capacidad del niño para expresar sentimientos, como afecto o cólera, en la forma y situaciones adecuadas. También evalúa los componentes afectivos de la conducta: sentimiento y actitudes del niño en diferentes ambientes como: durante el trabajo, en los juegos, en la escuela o en una situación nueva.

c) Autoconcepto: Evalúa el desarrollo de la conciencia y el conocimiento que el niño tiene de sí mismo: reconocimiento de su yo físico, sentimientos, intereses y preferencias., en los que se aprecian los sentimientos de autoestima en diversas situaciones.

d) Interacción con los compañeros: Evalúa la calidad y frecuencia de las interacciones del niño con sus compañeros de la misma edad, incluyendo: la capacidad para 
hacer amistades y establecer relaciones personales, responder e iniciar contactos sociales con los compañeros, interactuar eficazmente en un grupo pequeño y cooperar.

e) Colaboración: Evalúa la capacidad del niño para enfrentarse al entorno de forma eficaz, ya sea tolerando frustraciones, superando agresiones de compañeros, obedeciendo, resolviendo problemas o adaptándose a las normas.

f) Rol social: Evalúa tres aspectos del desarrollo social del niño/a:

- La capacidad para reconocer que niños y adultos tienen papeles distintos en situaciones diferentes y determinar qué es lo que se espera de ellos en cada situación;

- La capacidad para comprender las razones por las que se adoptan algunos comportamientos sociales. Por ejemplo, cooperación, ayuda, honradez, distinción entre lo que está bien y lo que está mal, sinceridad y otras conductas características del desarrollo ético y la capacidad para percibir y aceptar diferencias entre sí mismo y los demás.

- Comprender los puntos de vista, percepciones y sentimientos de los demás y mostrar solidaridad hacia ellos.

Las causas de adultos con problemas para socializar se generan en su mayoría durante la infancia, tanto en el entorno familiar, como en el ámbito escolar. Algunas de esas causas pueden ser: que los niños hayan sido separados de su familia, maltrato físico y emocional, timidez, falta de participación de los padres en su educación, burlas por parte de su padres y/o de sus iguales, amenazas, críticas destructivas, ambiente familiar y escolar de represión, entre otros (Papalia, 2005).

Por otro lado, en el interaccionismo se afirma que los rasgos de personalidad y las situaciones interactúan para influir en la conducta. Ni los ambientes ni la persona tomados por separado dan una explicación completa (Ekehammer, 1974; Endler y Magnusson, 1976; Magnusson y Endler, 1977; Ozer, 1986; Pervin, 1985; Snyder e Ickes, 1985).

Otra forma de definir las interacciones consiste en afirmar que algunas situaciones permiten la fácil expresión de la personalidad, mientras que otras la restringen a canales específicos e impiden su expresión (Monson, Hesley y Chernik, 1982; Shutte, Kenrick y Sadalla, 1985).

En la teoría del desarrollo cognitivo de Piaget se afirma que una de las principales características de la etapa de pensamiento preoperacional, en la que ubica a los niños de dos a siete años, es la "centración" o tendencia a enfocarse en un aspecto de una situación, e ignorar otros, siendo así que los preescolares llegan a conclusiones ilógicas porque no pueden descentrar o pensar en varios aspectos de una situación al mismo 
tiempo, y que esa centración limita el pensamiento de los niños de corta edad, sobre las relaciones físicas y sociales (Papalia, 2005).

Berruezo (2001), basado en una visión global de la persona, afirma que el término "psicomotricidad" integra las interacciones cognitivas, emocionales, simbólicas y motrices en la capacidad de ser y expresarse en un contexto psicosocial. La psicomotricidad así definida, desempeña un papel fundamental en el desarrollo armónico de la personalidad.

Se han observado que algunos aspectos de la personalidad pueden permanecer relativamente consistentes por largos periodos y esta estabilidad parece ser incluso mayor durante los años adultos que antes (Costa y McCrae, 1988, 1989; McCrae, 1993).

Picq y Vayer (1984) mencionan que la educación psicomotriz es una acción psicológica que utiliza los medios de la educación física con el fin de mejorar o normalizar el comportamiento del individuo (niño, niña, joven, etc.). Su pretensión es la de educar sistemáticamente las conductas perceptivo-motrices del alumno con objeto de facilitarle los diversos aprendizajes y la integración escolar y social.

En tanto, Le Boulch (1972) afirma que: "El dominio corporal es el primer elemento del dominio del comportamiento". Tan así, que de acuerdo a la neuropsicología y la psicofisiología, las cualidades fundamentales de la persona y su ajuste adecuado al medio, es posible utilizando el movimiento como recurso o sistema educativo.

A su vez, que se circunscribe a las técnicas psicomotrices en un entorno centrado en lo psicosomático y la comunicación, definiendo a la psicomotricidad, según García y Fernández, como la técnica o conjunto de técnicas que tienden a influir en el acto intencional y significativo, para estimularlo o modificarlo, utilizando como mediadores la actividad corporal y su expresión simbólica (González y González, 2006).

Acouturier (2007) plantea que muchos de los problemas localizados a nivel escolar, se nutren de situaciones de identidad y comunicación mal elaboradas, siendo el objetivo de la psicomotricidad, por consiguiente, el aumentar la capacidad de interacción del sujeto con el entorno.

Se realizó un estudio longitudinal centrado en la timidez y el temperamento infantil, que inició con madres de niños de 8 a 10 años de edad que hablaban de cómo eran sus hijos, posteriormente se entrevistó a los hijos cuando tenían 30 y 40 años y vivían con sus cónyuges y sus propios hijos. Las entrevistas revelaron que los chicos que en la niñez hacían berrinches frecuentes, al crecer eran hombres malhumorados; las niñas con pataletas constantes no se veían así al ser entrevistadas como adultas, pero sus familias las veían como madres malhumoradas. Los efectos de la timidez infantil también se presentaron en la vida posterior. Los niños tímidos se casaban más tarde y eran más lentos para establecer su carrera que los menos tímidos, las niñas tímidas no tardaban más en casarse, pero era más probable que siguieran un patrón convencional de madre de familia y de ama de casa que las menos tímidas (Caspi, Elder y Bem, 1987). 
Existen planes de adiestramiento para niños en edad prescolar y escolar. En ellos, los niños clasificados como aislados o marginados reciben una serie de sesiones que pueden incluir demostraciones de habilidades sociales específicas, oportunidades para practicarlas y refuerzo de su realización. Al menos en algunos casos estos planes han logrado aumentar de forma notable la aceptación social de niños que inicialmente se hallaban aislados (Rubin, 1989).

La psicomotricidad integra aspectos como la orientación temporal que es actuar corporalmente en consecuencia a un antes y un después; la estructuración temporal es la conciencia de los movimientos y de sus desplazamientos ejecutados en cierto tiempo y distancia y las nociones temporales como la designación del tiempo, por ejemplo lento-rápido, existe también mayor capacidad para modular su excitación emocional, por ejemplo controlar arrebatos de enfado, donde el adulto, ya sean los padres o la maestra de grupo, pueden ayudar al niño a maximizar ese control (Durivage, 1984).

En la teoría de desarrollo psicosocial de Erickson se afirma que: "cuantas más crisis se resuelvan de manera satisfactoria, mayor será su desarrollo" (Hopkins, 2000).

Por otro lado, la relación con los demás significa manejar emociones de forma socialmente aceptable, una manera de ayudarles a comprender sus emociones es hacerles hablar sobre ellas, tanto de las suyas como de las de los otros (Kuelbi, 1994).

Algunas estrategias que se recomiendan para mejorar el área personal/social son asegurarse que el clima de la clase fomente la expresión emocional, que los niños se sientan cómodos; estructurar el entorno físico para que el niño aprenda sobre sus sentimientos, utilizando materiales como marionetas para representar sus emociones (Sandtrock, 2003).

Las técnicas psicomotrices señaladas por García y Fernández, se circunscriben en un entorno centrado en lo psicosomático y la comunicación. Definen a la psicomotricidad como la técnica o conjunto de técnicas que tienden a influir en el acto intencional y significativo, para estimularlo o modificarlo, utilizando como mediadores la actividad corporal y su expresión simbólica; siendo el objetivo de la psicomotricidad, por consiguiente, el aumentar la capacidad de interacción del sujeto con el entorno (González y González, 2006).

Las competencias que componen el campo formativo personal y social, se favorecen en los pequeños a partir del conjunto de experiencias que viven y a través de las relaciones afectivas que tienen lugar en el aula y que deben crear un clima favorable para su desarrollo integral, ya que este representa una contribución fundamental para propiciar el bienestar emocional, aspecto fundamental en la formación de disposiciones para el aprendizaje en los alumnos" SEP (2004).

"La seguridad emocional que desarrollen los niños es condición fundamental para lograr una exploración más efectiva de las oportunidades de aprendizaje. La interpretación que podamos dar a las fallas en el aprendizaje de los niños debe reflexionarse vinculada a su sentimiento de seguridad, el cual puede expresarse en dificultades para 
relacionarse, bloqueo, aislamiento, falta de atención y concentración, y agresividad" SEP (2004).

Con base en las afirmaciones de los autores expertos en áreas psicomotriz y psicosocial y educativa, contenidas en el marco teórico, se realiza el planteamiento de este estudio pre experimental con el objetivo de observar si la intervención a través de un programa psicomotriz, podría influir en los niños de tercer grado de preescolar mejorando los aspectos de su área personal/social.

\section{Metodología}

Participantes

Un grupo de 29 niños de cinco años pertenecientes a tercer grado del preescolar México 91 ubicado en la Ciudad de Chihuahua, Chihuahua, México, siendo 13 mujeres y 16 hombres.

\section{Diseño}

Es un estudio pre experimental, en el cual se realizó una evaluación pre-post del área personal/social del Inventario de Desarrollo Battelle; El programa psicomotor aplicado duró 3 meses, realizándose 36 sesiones, administradas 3 veces por semana, de 45 minutos cada una. Se utilizó material reciclado y música de fondo.

\section{Instrumentos}

Para la medición fue utilizado el Inventario de Desarrollo BATTELLE.

Newborg, Stock y Wnek (1984) crearon el Inventario de Desarrollo Battelle, con una adaptación española posteriormente por De la Cruz y González. Se trata de una batería para evaluar las habilidades fundamentales del desarrollo en niños comprendidos entre el nacimiento y los ocho años, su aplicación es individual y está tipificada. Esta prueba, elaborada por un grupo de profesionales de diversos campos, se desarrolló inicialmente en el año 1984, aunque la adaptación española es del año 1996. Uno de los propósitos fundamentales con el que se creó la prueba fue el de poder proporcionar información sobre los puntos fuertes y débiles en diversas áreas del desarrollo del niño, para facilitar la elaboración de programas de intervención individualizados. Se utiliza en el ámbito clínico, en la evaluación en edades tempranas y en el área de la educación especial.

Es un inventario fácil de aplicar, que basa sus resultados en varias fuentes ya que los procedimientos para la obtención de datos son la observación, el examen estructurado e información que recoge de personas relevantes en la vida del niño. Su formato 
de aplicación y puntuación poseen mayor objetividad y rigor que los utilizados en la mayoría de los inventarios de desarrollo.

Los ítems se presentan en un formato normalizado que especifica la conducta que se va a evaluar, los materiales necesarios, los procedimientos de administración y los criterios para puntuar la respuesta. Está compuesto por 341 ítems divididos en las siguientes áreas:

- Personal/Social.

- Adaptativa.

- Motora (Motricidad Gruesa y Motricidad Fina).

- Comunicación (Receptiva y Expresiva).

- Cognitiva.

Dentro de cada una de las cinco áreas, los ítems se agrupan en subáreas (o áreas de habilidades específicas). Se utilizó un cuadernillo de aplicación, una hoja de respuestas, un lápiz y un borrador, el aplicador realizó las preguntas del área personal/social a cada uno de los niños en un tiempo aproximado de 15 minutos, también se realizaron entrevistas a la maestra sobre el comportamiento de cada niño, así como a los padres de familia, antes y después de la aplicación del programa.

Para realizar la revisión se emplearon las tablas estandarizadas por edad incluidas en el manual del inventario y los resultados en números naturales pasaron a convertirse en edades equivalentes de desarrollo de las cinco áreas, se transformaron posteriormente en cocientes de desarrollo, los cuales permitieron comparar los resultados obtenidos diferenciando la edad cronológica, la edad mental de desarrollo de cada una de las cinco áreas, así como la edad equivalente de desarrollo inter áreas o integral. Esta facilidad para realizar comparaciones de los tipos de edades fue el motivo que consideramos más atractivo para realizar la discriminación con otros tests que también manejan áreas personales y sociales, por el hecho de que obtenemos resultados de comparación, axiales y multiaxiales en relación al área de interés de este estudio.

Para la Intervención se diseñó el programa psicomotriz "Jugando en mi jardín":

La característica principal de este programa es que fue diseñado para mejorar los las áreas que se integran en el desarrollo del niño en edad de preescolar, por medio de actividades que estimulan aspectos psicomotrices como: tono muscular, orientación espacial y temporal, lateralidad, motricidad fina y gruesa, equilibrio y ritmo. Cada sesión constó de tres etapas: la etapa inicial con una duración cinco minutos y era acompañada de música rítmica infantil; la etapa medular con una duración de 30 minutos constaba de dos actividades motrices guiadas, dónde se utilizó el material de apoyo reciclado y económico: botellas y bolsas de plástico, cajas, botes, cartón, pelotas, aros, tela, con los que se diseñaron muñecos, cometas, boliche; finalmente la etapa de relajación con duración de $10 \mathrm{~min}$, en la que se les permitía descansar, se les sentaba en círculo y se les solicitaba comentaran sus apreciaciones sobre la sesión. 
Los métodos a considerar se incluyeron tanto de manera global, como por separado, en cada sesión contando con: asignación de tareas, exploración y solución de problemas, y aprendizaje a través de la experiencia.

El programa consta de 36 sesiones de juego repartidas en 8 semanas, impartiéndose tres veces por semana, con una duración de 45 minutos aproximadamente cada una.

\section{Procedimiento}

- Se compiló y reparó el material que previamente se había diseñado, para identificar el impacto de la psicomotricidad en el área personal/social de niños en educación preescolar para ser aplicado durante horario escolar a los/as alumnos/as de la escuela contemplada;

- Se programó una reunión con autoridades correspondientes de SEECH, para la presentación del proyecto con la finalidad de obtener la autorización correspondiente para acudir a la institución educativa donde se aplicaría el programa psicomotriz y el área personal/social del Inventario de desarrollo Batelle;

- Se programó una reunión con los directivos y los padres de familia, de la escuela preescolar de aplicación exponiendo la finalidad del estudio, las características y requerimientos del mismo;

- Una vez aceptada la propuesta, se solicitó de manera oficial, la respectiva autorización de los padres de familia;

- Se gestionó y capacitó a los recursos humanos necesarios para la aplicación;

- Se realizó la aplicación del área de la escala valorativa seleccionada en apoyo a la investigación, en dos etapas, diagnóstica y final, con el objetivo de contrastar resultados;

- Se aplicó el modelo psicomotriz a un grupo experimental, realizando 36 sesiones de Intervención con apoyo en música de fondo y el material didáctico diseñado para tal fin;

- Se manejaron los datos obtenidos por medio de estadística descriptiva y prueba $t$ de Student, análisis de varianza y regresión lineal para mediciones pre y post;

- Análisis de resultados para la emisión de una conclusión final y justificación de la propuesta de aplicación del modelo de intervención psicomotriz en educación preescolar. 


\section{Resultados}

Los datos obtenidos en las mediciones uno y dos de la Prueba Battelle en el área personal/social (tabla 1). Donde en la primera medición se observa una diferencia mayor a 11 puntos inclinada hacia la edad cronológica respecto a la edad equivalente, variando en la segunda medición donde la diferencia resulta menor a siete puntos.

De entre la medición uno y la medición dos (Tabla 2), en el subaspecto interacción con el adulto se obtuvieron cambios estadísticamente significativos $(\mathrm{p}=.029)$. En cuanto a expresión de sentimientos no se observan cambios significativos de $p=.143$. En el autoconcepto se observan diferencias significativas $(\mathrm{p}=.002)$, en cuanto a interacción con los compañeros la diferencia es significativa de $\mathrm{p}=.016$. En la colaboración se aprecian de igual manera cambios significativos (.000), obteniéndolos así mismo en el rol social (.007).

En la comparación de la medición uno con la medición dos (figura 1), con base en los resultados totales del área personal/social se muestra una correlación lineal estadísticamente significativa entre la edad cronológica inicial y de igual manera en la edad equivalente de desarrollo.

Se presentan los resultados promedio de las edades cronológicas y equivalentes de desarrollo del grupo en las mediciones uno y dos, mostrándose una correlación lineal significativa entre ellas (figura 2$),(r=.072 ; r 2=.489 ; \mathrm{P}=.000)$.

\begin{tabular}{|lllllc|}
\hline Medición 1 & N & Edad Cronológica 1 & Edad Equivalente 1 & niños & Niñas \\
\hline & \multirow{2}{*}{29} & Media \pm DE & Media \pm DE & \multirow{2}{*}{13} & \\
& & $62.59 \pm 3.56$ & $50.78 \pm$ DE 5.73 & & \\
\hline Medición 2 & $\mathbf{N}$ & Edad Cronológica 2 & Edad Equivalente 2 & niños & Niñas \\
& \multirow{2}{*}{29} & Media \pm DE & Media \pm DE & \multirow{2}{*}{13} & 16 \\
& & $64.86 \pm$ DE 3.35 & $58.50 \pm$ DE 5.13 & & \\
\hline
\end{tabular}

Tabla 1. Se muestran las diferencias de medias obtenidas en las mediciones uno y dos de la Prueba Battelle en el área personal/social.

\begin{tabular}{|c|c|c|c|c|c|c|}
\hline & $\begin{array}{c}\text { Interacción } \\
\text { con el } \\
\text { Adulto }\end{array}$ & $\begin{array}{c}\text { Expresión de } \\
\text { Sentimientos }\end{array}$ & Autoconcepto & $\begin{array}{c}\text { Interacción } \\
\text { con los } \\
\text { Compañeros }\end{array}$ & Colaboración & Rol Social \\
\hline $\begin{array}{c}\text { Medición } \\
\mathbf{1}\end{array}$ & $33.90 \pm 1.95$ & $22.41 \pm 1.80$ & $25.24 \pm 2.08$ & $27.14 \pm 4.11$ & $13.62 \pm 2.34$ & $21.97 \pm 4.19$ \\
\hline $\begin{array}{c}\text { Medición } \\
\mathbf{2}\end{array}$ & $34.93 \pm 1.00$ & $23.14 \pm 1.36$ & $26.86 \pm 1.30$ & $30.00 \pm 2.99$ & $16.48 \pm 1.99$ & $24.97 \pm 2.57$ \\
\hline $\mathbf{P}$ & 0.029 & 0.143 & 0.002 & 0.016 & 0.000 & 0.007 \\
\hline
\end{tabular}

Tabla 2. Se muestran los resultados de la media y desviación estándar obtenidos en cada sub aspecto del área personal/social, resultando positivos en la expresión de sentimientos y significativos en los demás comparando la medición uno y la medición dos. 
$R=.072 \quad R^{2}=.489 \quad P=.000$

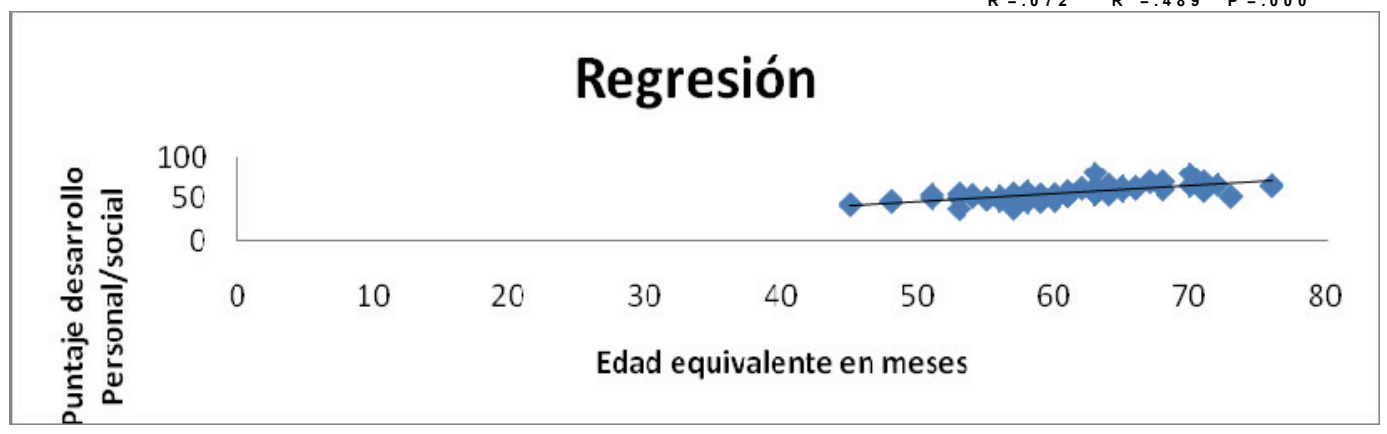

Figura 1. Se presentan los resultados promedio de las edades cronológicas y equivalentes de desarrollo del grupo en las mediciones uno y dos, mostrándose una correlación lineal significativa entre ellas.

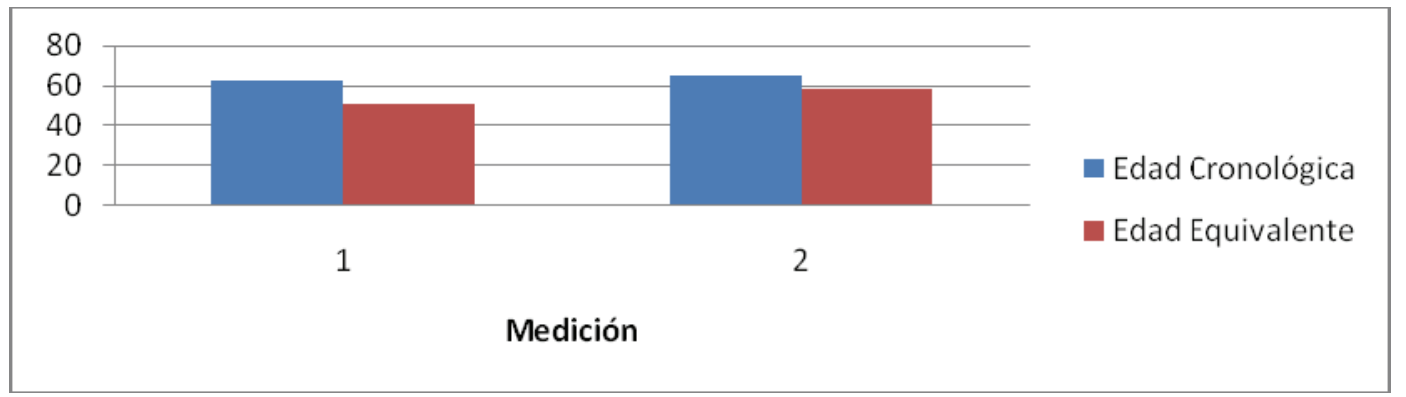

Figura 2. En la comparación de la medición uno con la medición dos, con base en los resultados totales del área personal/social se muestra una correlación lineal estadísticamente significativa entre la edad cronológica inicial y de igual manera en la edad equivalente de desarrollo.

\section{Discusión y conclusiones}

En el aspecto de Autoconcepto, se mostraron cambios estadísticamente significativos en cuanto al reconocimiento de su yo físico, sentimientos, intereses y preferencias., en los que se aprecian los sentimientos de autoestima en diversas situaciones. Aucouturier afirma que "la práctica psicomotriz educativa busca favorecer el desarrollo de la expresividad psicomotriz del niño para conseguir la comunicación, la creación y el pensamiento operatorio, entre los tres y los ocho años" (González y González, 2006). Por lo que se considera que posiblemente haya una correlación positiva entre el cambio y la influencia del programa psicomotriz.

En relación a la Interacción con los compañeros, la capacidad para hacer amistades y establecer relaciones personales, responder e iniciar contactos sociales con los compañeros, interactuar eficazmente en un grupo pequeño y cooperar se vieron afectadas de manera positiva, lo que concuerda con la afirmación que se ha planteado de que "muchos de los problemas localizados a nivel escolar, se nutren de situaciones de iden- 
tidad y comunicación mal elaboradas, siendo el objetivo de la psicomotricidad, por consiguiente, el aumentar la capacidad de interacción del sujeto con el entorno" (Acouturier, 2004).

Respecto al subaspecto de Colaboración, se observó de manera general mayor tolerancia a la frustración por parte de los niños, superando agresiones de compañeros, obedeciendo, resolviendo problemas y adaptándose a las normas con mayor frecuencia. Lo que se considera que puede estar relacionado con la afirmación de Chokler: "la psicomotricidad es la disciplina que estudia al hombre, descodificando el campo de significaciones que el cuerpo y el movimiento en relación generan y que constituyen señales de su desarrollo y sus posibilidades de aprendizaje e inserción social" (González y González, 2006).

En relación al Rol Social, se observó que aumentó significativamente en los niños la capacidad para reconocer que niños y adultos tienen papeles distintos en situaciones diferentes y la determinación de qué es lo que se espera de ellos en cada situación. Así mismo la capacidad para comprender las razones por las que se adoptan algunos comportamientos sociales, como la cooperación, ayuda, honradez, distinción entre lo que está bien y lo que está mal, sinceridad y otras conductas características del desarrollo ético. La capacidad para percibir y aceptar diferencias entre sí mismo y los demás, mayor comprensión, en general, de los puntos de vista, percepciones y sentimientos de los demás y un aumento en la solidaridad hacia ellos. Lo que posiblemente sea explicado por lo descrito en la teoría cognitiva socio-cultural de Vigotsky, la cual afirma que las habilidades cognitivas tienen su origen en las relaciones sociales y culturales, considerando la visión de que el conocimiento es situado y colaborativo, es decir, que se distribuye entre la gente y el entorno, incluyendo objetos, herramientas, libros y la comunidad en que se vive. Lo que sugiere que el conocimiento avanza más a través de la interacción con los demás en las actividades que requieren cooperación (Kosulin, 2000).

La relación con el cambio observado en los resultados, puede ser explicada por el hecho de circunscribirse a las técnicas psicomotrices dentro de un entorno centrado en lo psicosomático y la comunicación. Definiendo a la psicomotricidad como la técnica o conjunto de técnicas que tienden a influir en el acto intencional y significativo, para estimularlo o modificarlo, utilizando como mediadores la actividad corporal y su expresión simbólica (González y González, 2006). Por lo cual se considera que el programa psicomotriz puede haber tenido influencia directa para el desarrollo de dichos subaspectos.

Dentro los subaspectos ya mencionados, se observaron modificaciones positivas en las interacciones que incluyen comportamientos como el apego infantil, la respuesta al inicio de contactos sociales y la utilización de los adultos como recurso. Tomando en cuenta la afirmación de que "la psicomotricidad es relevante para distinguir la motricidad humana de la animal, pues implica en su conjunto una función intencional modificadora y un origen social; por intermediación del psiquismo el movimiento se 
convierte en un gesto con respuesta, intencionalidad y significación" (Da Fonseca, 1998). Se considera que el desarrollo y la magnitud alcanzada puede ser explicada tanto por la intervención del programa, como por variables extrañas como el tiempo y ambiente familiar.

En cuanto a Expresión de sentimientos/afecto, que es la capacidad para expresarlos en la forma y situaciones adecuadas, se observaron cambios de actitud en la mayoría de los niños, tanto intra como interpersonales. Sin embargo, para este aspecto los resultados no fueron estadísticamente significativos, lo cual podría explicarse por la amplitud del tiempo en que se aplicó la intervención psicomotriz, siendo esta considerada corta. Ya que en la teoría del desarrollo cognitivo de Piaget se afirma que una de las principales características de la etapa de pensamiento preoperacional, en la que ubica a los niños de dos a siete años, es la "centración" o tendencia a enfocarse en un aspecto de una situación, e ignorar otros. Siendo así que los preescolares llegan a conclusiones ilógicas porque aún no pueden descentrar o pensar en varios aspectos de una situación al mismo tiempo, y esa centración limita el pensamiento de los niños de corta edad, sobre las relaciones físicas y sociales (Papalia, 2005). Para contrarrestar estos aspectos, se considera necesario aumentar el tiempo de intervención psicomotriz, considerando como mínimo, el lapso que engloba la etapa de maduración, para observar la generación del cambio en su proceso natural.

Es de suma importancia que la escuela brinde oportunidades de encuentro con otros niños a través de las actividades lúdicas, porque es bien reconocido que en esta etapa del esquema conceptual, al niño le agrada jugar y estar con otros niños del mismo o diferente género, sobre todo cuando empieza este segundo periodo (García, 1992).

Por otra parte se afirma que cuanto más intensa es una frustración, más posible es la agresión, ya que los niños son dependientes y esto da a entender un desarrollo social que se da en un dominio gradual de agresión que toma una forma socialmente aceptable, dinámica que debe darse con elementos y situaciones que para el niño sean gratificantes y significativos, porque de esta forma se estará contribuyendo al desarrollo social, en la medida en que los estímulos que le llegan sean acordes con una situación gratificante como el juego (Musen, Conger y Kagan (1979).

Las frustraciones que los niños tienen en su quehacer son las propias y lógicas de las actividades propuestas. Si las circunstancias se propician de esta manera, García afirma que la agresión resulta mínima y existe un estímulo-respuesta gratificante, que repercute en forma directa con el desarrollo social (García, 1992).

Se observó que la edad equivalente, que representa la edad integral de la totalidad de áreas de desarrollo guarda una estrecha relación con el desarrollo del área personal/social y que el aumento de una afecta directamente a la otra.

Con base en los resultados obtenidos a través de esta investigación donde se observa claramente una mejoría en el área personal/social de los niños en quienes fue aplicado el programa, se concluye que la intervención psicomotriz pudo haber sido un 
factor importante en el impacto positivo y significativo obtenido, no obstante se reconoce que el tiempo de aplicación fue corto, siendo posiblemente insuficiente para obtener resultados más significativos, por lo que se recomienda continuar con investigaciones en el área de estudio utilizando programas de intervención psicomotriz durante la totalidad del periodo escolar, lo que podría redundar en cambios más favorables e inclusive relativamente permanentes.

\section{Referencias bibliográficas}

AUCOUTURIER, B. (2004) Los fantasmas de la acción y la práctica psicomotriz. España: GRAO de IKIF S, L.

BERRUEZO, P. (2001). El contenido de la psicomotricidad. Reflexiones para la delimitación de su ámbito teórico y práctico. Revista iberoamericana de psicomotricidad y técnicas corporales, (1), 39-48.

CASPI, A., ELDER, G. H., JR., \& BEM, D.J. (1987). Moving against the world: Lifecourse patterns of explosive children. Developmental Psychology, 23, 308-313.

COSTA, P. T., JR., \& MCCRAE, R. R. (1988). Personality in adulthood: A six-year longitudinal study of self-reports and spouse ratings on the NEO personality inventory. Journal of Personality and Social Psychology, 54, 853-863.

COSTA, P. T., JR., \& MCCRAE, R. R. (1989). Personality continuity and the changes of adult life. In M. Storandt \& G. R. VandenBos (Eds.), The adult years: Continuity and Change (pp. 45-77). Washington, DC: American Psychological Asosiation.

CRAVER, C., \& SHEIER, M. (1997). Teorías de la personalidad. (Tercera ed.p.4). MEXICO: Prentice Hall.

DA FONSECA, V. (1998). Manual de observación psicomotriz. (Pimera Ed.). España:INDE.

DURIVAGE, J. (2007). Educación y psicomotricidad: manual para el nivel preescolar. México: Trillas.

EKEHAMMER, B (1974). Interactionism in personality from a historical perspective. Psicological Bulletín, 81, 1026-1048.

ENDLER, N. S., \& MAGNUSSON, D. (1976). Interactional psychology and personality. Washington, D. C.:Hemisphere.

GARCÍA GÓMEZ, A. (1992). Una experiencia sobre la mejora del autoconcepto en el aula. En Varios. Experiencias de orientación en Educación Básica. (pp. 21-61). Madrid: MEC.

GONZÁLEZ, E, \& GONZÁLEZ, M. (2006). Reflexiones sobre la educación psicomotriz y su incidencia curricular en primaria. Valladolid: Centro de psicomotricidad y formación ocupacional psicovital. 
HOPKINS, J. R. (2000). Erikson, E.H. In A. Kazdin (Ed.), Enciclopedia of psychology. Washington, DC, \& New York: American Psychological Association and Oxford University Press.

KOSULIN, A. (2000). Vygotsky. In A. Kazdin (Ed.), Encyclopedia of Psychology. Washington, DC, \& New York: American Psychologycal Association and Oxford University Press.

KUELBI, J. (1994, March). Young children's understanding of everyday emotions. Young Children, pp. 22-33.

LE BOULCH, J. (1972) Le education Par le mouvement. Paris, ESF. Nuviéme edition.

MAGNUSSON, D., \& ENDLER, N.S. (Eds.). (1977). Personality at the crossroads: Current issues in international psychology. Hillsdale, NJ: Erlbaum.

MCCRAE, R. R. (1993). Moderated analyses of logitudinal personality stability. Journal of Personality and Social Psychology, 65, 577-585.

MONSON, T., HESLEY, J., \& CHERNICK, L. (1982). Specifying when personality traits can and cannot predict behavior: An alternative to abandoning the attempt to predict single-act criteria. Journal of Personality and Social Psychology, 43, 385399.

MUSSEN, CONGER \& KAGAN (1979). Desarrollo de la personalidad en el niño. México:Trillas.

NEWBORG,J., STOCK, WNEK, L., GUIDUBALDI, J. Y SVINICKI, J. (1984); Inventario de Desarrollo Battelle. Barcelona: Fundación Catalana per a la Síndrome de Down.

OZER, D.J. (1986). Consistency in personality: A methodological framework. New York: Springer-Verlag.

PAPALIA, D, WENDKOS, et al. (2005). Psicología del desarrollo de la infancia a la adolescencia. México: Mc Graw Hill.

PERVIN, L.A. (1985). Personality: Current controversies, issues, and directions. In M. R. Rosenzweig \& L. W. Porter (Eds.), Annual review of psychology (Vol. 36). Palo Alto, CA: Annual Reviews.

PICQ, L. ET P. VAYER (1984). Education psycho-motrice et arrierátion mentale. (4éme Ed.). Paris:Doin.

RUBIN, Z. (1989). Children frienships. Londres:Openbooks Publishing, LTD., pp 74.

SANDTROK, J. (2003). Psicología del desarrollo en la infancia. España: Mc Graw Hill.

SEP. (2004). Programa de educación preescolar. México: Comisión Nacional de libros de texto gratuitos. 
SHUTTE, N.S., KENRICK, D.T., \& SADALLA, E. K. (1985). The search for predictable settings: Situational prototypes, constraint, and behavioral variation. Journal of Personality and Social Psychology, 49, 121-128.

SNYDER, M. \& ICKES, W. (1985). Personality and social behavior. In G. Lindzey \& E. Aronson (Eds.), Handbook of social psychology (3rd ed.). Vol II: Special fields and applications. New York: Random House.

ZABALZA, M. (2006). Didáctica de la educación infantil. Madrid: Narcea, S.A. de Ediciones.

Correspondencia con los autores

Rocío Rubio Ponce; David Arnoldo García Fernández; Natanael Cervantes Hernández

Facultad de Educación Física y Ciencias del Deporte

Universidad Autónoma de Chihuahua

e-mail:xio123@hotmail.com 